\title{
Three-dimensional Reconstruction and Measurement of Avian Eggs through Digital Imaging
}

\author{
Wasif Shafaet Chowdhury, Gang Lu, Md Moinul Hossain \\ School of Engineering and Digital Arts, University of Kent, Canterbury, Kent CT2 7NT, UK \\ wasif.shafaet.chowdhury@g.bracu.ac.bd,g.lu@kent.ac.uk,m.hossain@kent.ac.uk
}

\begin{abstract}
This paper presents a computer vision-based method for the 3-D (three-dimensional) reconstruction and characterization of avian eggs. Two low-cost cameras are used to acquire images of eggs from top and side views, respectively. The image segmentation is performed using the image binarization technique. The contour-slice based method is employed for the 3-D reconstruction. The geometrical parameters of avian eggs, such as length, breadth, volume and surface area, are then computed based on the reconstructed model. The performance of the system is evaluated using eggs from different breeds and sizes. Comparative results between the physical measurement and the proposed approach suggest that the digital imaging approach has an overall accuracy of $\mathbf{9 8 \%}$ for the geometrical parameter measurement of avian eggs.
\end{abstract}

Keywords - Avian eggs, Digital imaging, 3-D reconstruction, geometrical parameters

\section{INTRODUCTION}

There are around 1.1 trillion eggs that are produced per annum worldwide, among them, 40 million being incubated for reproductive purposes. It is therefore critical to monitor and maintain eggs in good traits, incubation, hatchability and storage conditions, particularly in high-productive commercial poultry lines. Due to a lack of effective on-line and real-time measurement techniques, however, the weight of the egg is the only parameter which is widely used for assessing the quality of eggs in the poultry industry $[1,2]$. Studies suggest that other geometric parameters, such as volume and surface area, are crucial for adjusting incubation regimes in industrial hatcheries $[1,2]$. Moreover, an avian egg is considered as a highly integrated biological system. Any abnormality within such a system can collapse its physiological functions that are necessary to provide the best environment for the development of an embryo [1]. It has been concluded that egg weight, shell properties, shape (length and breadth ratio), and egg contents are the most important parameters for successful hatching [2]. To increase hatchability and to assist the incubation process, the measurement and characterization of the egg are significant in both ecological and economical aspects.

There are a number of studies to establish the relationship between egg weight and hatchability. It is shown that the eggs of average weight $(50-60 \mathrm{~g})$ are more likely to have good hatchability $[3,4]$. Narushin et al. suggested that the ratio of the egg weight and the surface area can be used for a better estimation of hatchability [1]. Egg shape is another significant factor for the determination of egg hatchability. It is understood that the egg shape remains consistent during the whole incubation process. The ratio between the length and breadth can also be used as a spatial feature [1]. Various invasive techniques, such as traditional or modified Archimedes' water displacement methods, were used to determine the volume of the egg [5-7]. However, there is no direct method to measure accurately the surface area and volume of the egg. A technique to estimate the surface area is to cover the eggshell with tape strips, and then peel tape strips off and measure the area of the peeled strips [8]. This invasive method is error-prone, time-consuming and labor-intensive. Mathematical modeling approaches were also used to estimate the shape and volume of the eggs $[9,10]$. However, the shape of the egg varies largely, which introduces inaccuracies in the mathematical models of the egg.

Digital imaging and image processing are considered to be non-invasive techniques and thus have received great attention for measuring the physical properties of eggs $[8,11]$. For instance, 2-D (two-dimensional) image processing techniques were used to measure the maximum length and breadth of eggs [12-14]. In those methods, however, the egg images were captured without considering the tilted geometry problem of the egg [12]. The tilted geometry problem of the egg occurs since the egg yolk is heavier than the egg white. Troscianko et al. proposed a mathematical formula to estimate the shape of the egg. They extracted egg edge points manually from gridded background images to estimate the egg size and volume [13]. Zalhan et al. also used a digital imaging approach to measure the radius of the egg [14], where the pixel size of the egg images was estimated from reference values obtained through a coordinate measuring machine, which involves high levels of operational complexity. Very limited work was carried out for the 3-D reconstruction and characterization of avian eggs. Ting et al. [12] used a Microsoft Kinect imaging device for measuring the volume of egg, where a geometric model and 3-D point-cloud processing algorithm was used. Siswantoro et al. [15] used the Monte Carlo method for computing the volume of egg, where five images of the egg were acquired from five different views to approximate the volume of the egg. The diameters of the egg were manually determined and then fed into a regression equation for the volume estimation. The whole process is time-consuming and labor-intensive. Zhang et al. [16] proposed a 3-D method for computing the volume and surface area of an avian egg from multi-angle images. This method requires images from different positions and orientations for the photogrammetric bundle adjustment.

It is clear that, although various progress has been made, a 3-D and real-time method is still desirable for measuring and characterizing the geometrical parameters of avian eggs reliably and accurately. This paper presents a digital imaging based method for the 3-D reconstruction and characterization of avian eggs. Two low-cost cameras are used to acquire the images of eggs from top and side views, respectively. The RGB images are converted to the CMYK (Cyan, Magenta, Yellow, and Key) color space, and the Y-component images are used for binarization and later the segmentation of the egg region. An orientation correction procedure is taken to align the major axis of egg image with reference to the $x-y$ coordinate system, so that a bounding box object localization approach can be applied to determine the maximum length 
and breadth. A contour slice-based image processing algorithm is employed for the 3-D reconstruction of the egg. The performance of the proposed method is verified through a comparative analysis between the reference measurements and the obtained results. The test results are presented and discussed.

\section{Methodology}

\section{A. Image Acquisition}

Figures 1 and 2 illustrate the block diagram and physical implementation of the system, respectively. Two identical low-cost RGB cameras were placed at the top (Camera 1) and the side (Camera 2) inside a bespoke enclosure. The camera (Logitech C920 HD Pro) has a standard autofocus lens, and an image resolution of $1080(\mathrm{H}) \times 720(\mathrm{~V})$ at a frame rate of 30 frames per second. The images are transmitted to a computer. Application software with a GUI (Graphical User Interface) is developed to process images.

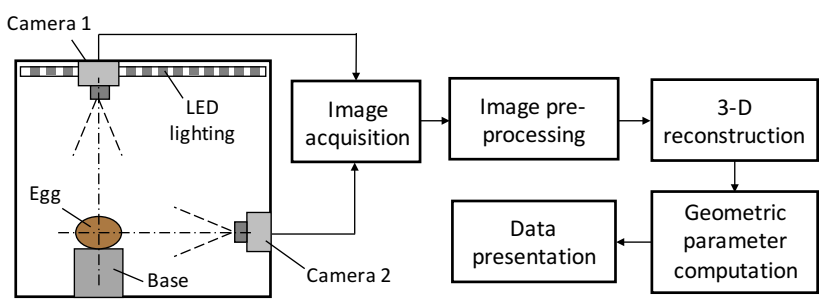

Fig. 1. Block diagram of the proposed methodology.

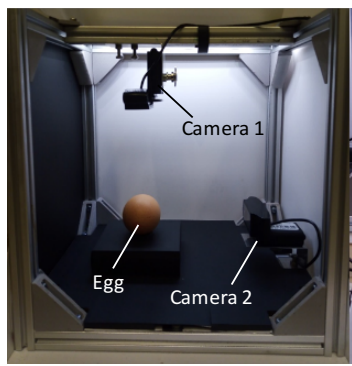

(a) Enclosure

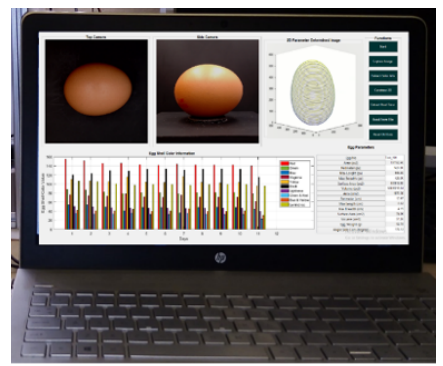

(b) Graphical User Interface
Fig. 2. Physical implementation of the system.

Camera 1 performs as a primary sensor, images acquired by which are served for measuring the geometrical parameters of eggs. Camera 2 is used as an auxiliary sensor, for correcting the possible misalignment of sample eggs in the measurement. Two dimmable LED (Light-emitting Diode) strips, each has 120 LEDs, were placed at the top of the enclosure to create a stable and uniform lighting condition for the image acquisition. To minimize the possible light reflection, all the inner surfaces of the enclosure were covered with nonreflective black paint.

The two cameras were carefully aligned so that the optical paths of the cameras met orthogonally at the center of the test egg (in the consideration of the averaged size of eggs, Fig. 1), and have the same distance to the center. This was done through a system calibration using an egg template the size (i.e., the major axis and the maximum breadth) of which was pre-measured and marked. The defined geometric center was then used to align the optical paths of the two cameras. A chessboard template of $20 \times 20 \mathrm{~mm}$ in size with $5 \times 5$ grids was also used to assess the perspective distortion of acquired images. It has been found that the system has an overall mean error of 0.37 pixel (only for an evaluation purpose) in the linear measurement. In addition, as the size of eggs varies, the objective distance of the camera to the egg can be different. Thus, an adaptive calibration factor, expressed as the number of pixels per centimeter, was introduced. This was performed only for Camera 1 by taking images of a standard ruler for 15 different levels with an interval of $0.3 \mathrm{~cm}$ along the camera optical path (level 'zero' is the base where eggs lie). The relationship of the absolute dimension and the number of pixels can then be established for each level, which is expressed by a fitting curve, i.e.,

$$
f_{c}=0.69 x+100.9
$$

where $f_{c}$ is the adaptive calibration factor (pixels/cm), $x$ is the distance $(\mathrm{cm})$ from a point along the optical path of Camera 1 , ranging between 'zero' (the level of the base) and the maximum breadth $\left(B_{m}, \mathrm{~cm}\right)$.

\section{B. Image Pre-processing}

1) Binarization and segmentation- The eggs are different not only in size but also in color. The conventional binarization techniques are therefore ineffective. In this study, the RGB images were first converted to the CMYK color space as it was found that the yellow color component (Ycomponent) in this color space can provide the complete region of eggs with different colors. Fig. 3 represents the original image, Y-component image and binarized image of an egg, respectively. The binarized image (segmentation) was obtained using a threshold value of 0.1 which was determined through a trial and error approach.

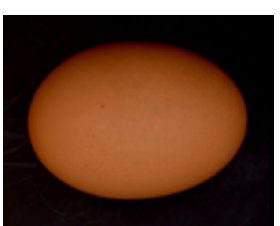

(a)

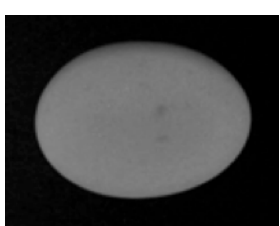

(b)

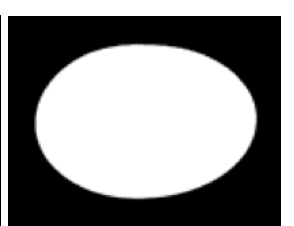

(c)
Fig. 3. Binarization and segmentation: (a) Original image; (b) Y-component image; and (c) Binarized image.

2) Orientation correction- There is a possibility that the major axis of the egg imaged (by Camera 1) is not in parallel with the $\mathrm{x}$-axis of the image coordinate system which can introduce difficulties in the computation of the major axis and breadth, as well as the 3-D reconstruction of the egg. Hence, a step needs to be taken to correct the orientation of the acquired egg images. This is done by determining first the geometric center of the egg image. For a binarized egg image, its geometric center is to coincide with the centroid of the image, i.e., $C\left(x_{c}, y_{c}\right)$ as shown in Fig. 4(a), and we have,

$$
\left\{\begin{array}{l}
x_{c}=\frac{\sum x_{p i} p_{i}}{\sum p_{i}}, \\
y_{c}=\frac{\sum y_{p i} p_{i}}{\sum p_{i}},
\end{array}\right.
$$

where $x_{p i}$ and $y_{p i}$ are the $\mathrm{x}$ - and $\mathrm{y}$-coordinates of pixel $p_{i}$ and $i$ belongs to the image region of the egg.

Once the centroid $(C)$ is allocated, the distance $(r)$ from $C$ to a point on the edge of the egg, $p(x, y)$, can be calculated by,

$$
r=\sqrt{\left(x-x_{c}\right)^{2}-\left(y-y_{c}\right)^{2}} .
$$

The maximum $r\left(r_{\max }\right.$ as shown in Fig. 4(b)) represents the major-radius of the egg, and the angle between the major- 
radius and the $\mathrm{x}$-axis of the image coordinate system, $\theta$, can then be obtained. The image is finally rotated by $\theta$ degrees so that the major-axis (having the same orientation as the majorradius) is in parallel with the $\mathrm{x}$-axis of the image coordinate system, as illustrated in Fig. 4(c).

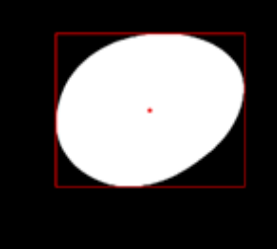

(a)

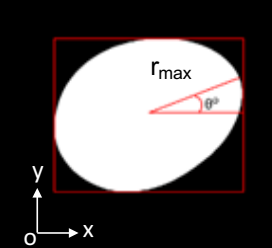

(b)

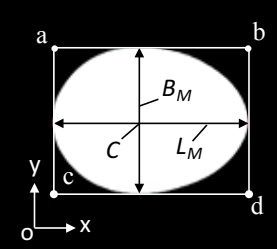

(c)
Fig. 4. Orientation correction: (a) Misaligned image; (b) Determined majorradius and $\theta$; (c) Image after the orientation correction and with the bounding box.

3) Maximum Length and Breadth-Once the orientation of the egg image is corrected, the bounding box can be used to localize the egg region by using the technique given in [17]. The bounding box is the tightest box containing the image region of the egg, as illustrated in Fig. 4(c). The maximum length $\left(L_{M}\right)$ and breadth $\left(B_{M}\right)$ of the egg are then defined as the length and breadth of the bounding box, respectively.

\section{3-D Reconstruction}

The 3-D reconstruction of the egg is based on the assumption that each breadthwise cross-section of the egg is circular, and the center of the cross-section lies on the major axis (the $\mathrm{z}$-axis of an $\mathrm{x}-\mathrm{y}-\mathrm{z}$ coordinate system, as shown in Fig. 5). This is true based on previous studies [18]. After the maximum length of the egg is computed, the radius of each cross-section can be determined by calculating the distance from the major-axis to the edge of that cross-section using Eq. (3).

Once all the radii of the cross-sections are computed, cross-sectional slices can be drawn along the maximum length, forming the 3-D model of the egg as shown in Fig. 6.

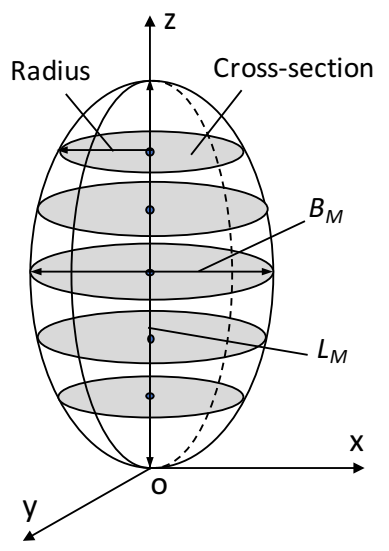

Fig. 5. 3-D model of an egg.

\section{Volume and Surface Area}

The volume $\left(V, \mathrm{~cm}^{3}\right)$ and surface area $\left(S, \mathrm{~cm}^{2}\right)$ of the egg are computed based on the reconstructed 3-D model by taking the accumulations of the areas and perimeters of all the crosssectional slices, respectively, i.e.,

$$
V=\frac{1}{{\overline{\mathrm{f}_{\mathrm{c}}}}^{3}} \sum_{1}^{N} A_{C S}
$$

$$
S=\frac{1}{\overline{\mathrm{f}}_{\mathrm{c}}^{2}} \sum_{1}^{N} P_{C S}
$$

where $A_{C S}$ is the area of a cross-section which equals to the total number of pixels within the cross-section. $P_{C S}$ is the perimeter of a cross-section which equals to the total number of pixels along the perimeter of the cross-section. $N$ is the total number of the cross-sections which is basically the maximum length of the egg (in pixel). $\overline{\mathrm{f}}_{\mathrm{c}}$ (pixels $/ \mathrm{cm}$ ) is the adaptive calibration factor as given in Eq. 1 where $x$ equals to a half of the maximum breadth (i.e., the central plane of the egg).

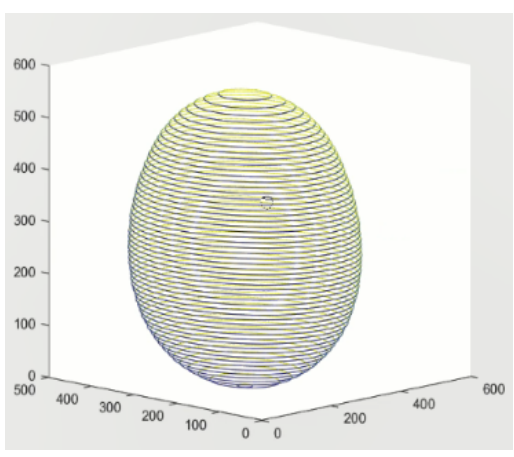

Fig. 6. Complete 3-D model of an egg.

\section{EXPERIMENTAL RESULTS AND DISCUSSION}

\section{A. Egg Samples}

In order to evaluate the performance of the proposed measurement system, a total of 24 fresh eggs with four different types and three different sizes were examined. Table I summaries the types and sizes of the eggs tested. Fig. 7 shows the example images of the eggs taken by the imaging system.

TABLE I. TYPES OF THE TESTED EGGS.

\begin{tabular}{|c|c|c|}
\hline Egg Type & Size & Quantity \\
\hline \multirow{2}{*}{ Free Range (FR) } & Medium $\left(\mathrm{M}_{\mathrm{f}}\right)$ & 4 \\
\cline { 2 - 3 } & Large $\left(\mathrm{L}_{\mathrm{f}}\right)$ & 4 \\
\cline { 2 - 3 } & Extra Large $\left(\mathrm{XL}_{\mathrm{f}}\right)$ & 4 \\
\hline Caged $(\mathrm{C})$ & Mixed Size $\left(\mathrm{M}_{\mathrm{C}}\right)$ & 4 \\
\hline Blue $(\mathrm{B})$ & Mixed Size $\left(\mathrm{M}_{\mathrm{B}}\right)$ & 4 \\
\hline Deep Brown $(\mathrm{DB})$ & Mixed Size $\left(\mathrm{M}_{\mathrm{D}}\right)$ & 4 \\
\hline
\end{tabular}

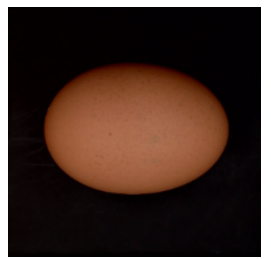

(a)

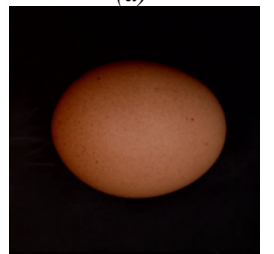

(d)

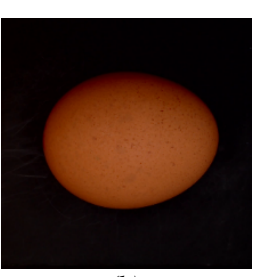

(b)

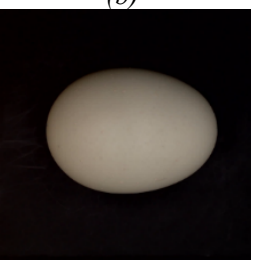

(e)

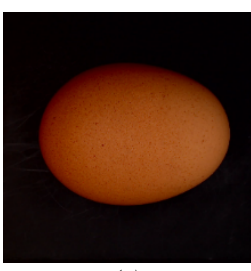

(c)

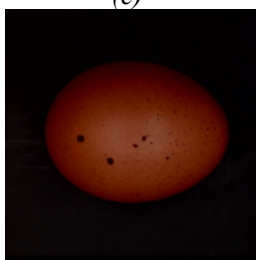

(f)
Fig. 7. Example egg images: (a) Free range $\left(\mathrm{M}_{\mathrm{f}}\right)$, (b) Free range $\left(\mathrm{L}_{\mathrm{f}}\right)$, (c) Free range $\left(X_{f}\right),(d)$ Caged $\left(M_{C}\right),(e)$ Blue $\left(M_{B}\right)$ and (f) Deep brown $\left(\mathrm{M}_{\mathrm{D}}\right)$. 
All the eggs were numbered and their geometric parameters including the maximum length and breadth were measured manually using a Vernier caliper with an accuracy of $0.01 \mathrm{~mm}$. The volume of each egg was also measured using the Hughes technique [19] in which the egg was suspended beneath the surface of the water in a container placed on an electronic scale with an accuracy of $0.01 \mathrm{~g}$. The volume of the egg is the increase in weight divided by the density of the fluid (i.e., water). The measured geometric parameters and volume of the eggs are given in Tables II and III.

TABLE II. MEASURED MAXIMUM LENGTH AND BREADTH

\begin{tabular}{|c|c|c|c|c|c|c|c|}
\hline \multirow[b]{2}{*}{$\begin{array}{c}\text { Egg } \\
\text { Type }\end{array}$} & \multirow[b]{2}{*}{ Size } & \multirow[b]{2}{*}{$\begin{array}{c}\mathbf{L}_{M_{P} P M} \\
(\mathbf{c m})\end{array}$} & \multirow[b]{2}{*}{$\begin{array}{c}\mathbf{L}_{M_{-} I M} \\
(\mathbf{c m})\end{array}$} & \multirow[b]{2}{*}{$\begin{array}{l}\text { RE } \\
(\%)\end{array}$} & \multicolumn{3}{|c|}{ Standard Deviation } \\
\hline & & & & & $\begin{array}{c}\mathbf{L}_{M_{-} \mathbf{P M}} \\
(\mathbf{c m})\end{array}$ & $\begin{array}{c}\mathbf{L}_{M_{-} I M} \\
(\mathbf{c m})\end{array}$ & $\begin{array}{l}\text { RE } \\
(\%)\end{array}$ \\
\hline \multirow{3}{*}{ FR } & $\mathrm{M}_{\mathrm{f}}$ & 5.63 & 5.70 & 1.12 & 0.15 & 0.13 & 0.16 \\
\hline & $\mathrm{L}_{\mathrm{f}}$ & 5.89 & 5.95 & 1.11 & 0.13 & 0.13 & 0.05 \\
\hline & $\mathrm{XL}_{\mathrm{f}}$ & 6.29 & 6.36 & 1.23 & 0.13 & 0.13 & 0.27 \\
\hline $\mathrm{C}$ & $\mathrm{M}_{\mathrm{C}}$ & 5.88 & 5.95 & 1.28 & 0.21 & 0.22 & 0.17 \\
\hline $\mathrm{B}$ & $\mathrm{M}_{\mathrm{B}}$ & 5.83 & 5.88 & 0.94 & 0.20 & 0.21 & 0.22 \\
\hline DB & $\mathrm{M}_{\mathrm{D}}$ & 6.00 & 6.04 & 0.78 & 0.24 & 0.27 & 0.23 \\
\hline $\begin{array}{c}\text { Egg } \\
\text { Type }\end{array}$ & Size & $\begin{array}{c}\mathbf{B}_{M_{-} P M} \\
(\mathbf{c m})\end{array}$ & $\begin{array}{c}\text { B }_{M_{L} I M} \\
(\mathbf{c m})\end{array}$ & $\begin{array}{c}\text { RE } \\
\%\end{array}$ & $\begin{array}{c}\mathbf{B}_{M_{-} P M} \\
(\mathbf{c m})\end{array}$ & $\begin{array}{c}\mathrm{B}_{\mathrm{M}_{-} \mathrm{IM}} \\
(\mathrm{cm})\end{array}$ & $\begin{array}{c}\text { RE } \\
\%\end{array}$ \\
\hline \multirow{3}{*}{ FR } & $\mathrm{M}_{\mathrm{f}}$ & 4.27 & 4.24 & -0.64 & 0.10 & 0.09 & 0.47 \\
\hline & $\mathrm{L}_{\mathrm{f}}$ & 4.43 & 4.38 & -1.12 & 0.13 & 0.11 & 0.27 \\
\hline & $\mathrm{XL}_{\mathrm{f}}$ & 4.80 & 4.76 & -0.74 & 0.11 & 0.13 & 0.41 \\
\hline $\mathrm{C}$ & $\mathrm{M}_{\mathrm{C}}$ & 4.46 & 4.45 & -0.17 & 0.09 & 0.10 & 0.37 \\
\hline $\mathrm{B}$ & $\mathrm{M}_{\mathrm{B}}$ & 4.31 & 4.30 & -0.17 & 0.15 & 0.15 & 0.15 \\
\hline DB & $\mathrm{M}_{\mathrm{D}}$ & 4.46 & 4.47 & 0.17 & 0.20 & 0.21 & 0.19 \\
\hline
\end{tabular}

\section{Results and Discussion}

Table II illustrates the average maximum length and breadth obtained through the physical measurement (PM) and the imaging technique (IM). The relative error (RE) and the standard deviation are also presented. It can be seen that the measured length and breadth are very close to the physical measurements. Higher deviations can be seen for the C, B and DB eggs in comparison with the FR eggs in both the physical and proposed measurements. It occurred because these eggs are of mixed sizes, In addition, the measured length and breadth of the DB eggs have the highest deviation among the tested eggs. For the length measurements, the $\mathrm{C}$ eggs have the maximum relative error of $1.28 \%$, and the DB eggs have the smallest error of $0.78 \%$. It is clear that the proposed technique is capable of determining accurately the length and breadth of the eggs.

Table III summarizes the average volumes of the eggs measured physically as well as through digital imaging, whilst Table IV gives the surface-areas computed based on the images. It can be seen that the measured volumes $\left(\mathrm{V}_{\mathrm{PM}}\right)$ and computed ones through imaging $\left(\mathrm{V}_{-} \mathrm{IM}\right)$ are very similar and the maximum relative error no more than $0.5 \%$. The maximum deviation of $7.15 \mathrm{~cm}^{3}$ is observed for the DB eggs, attributed to their size variation (note the DB eggs are of mixed size). It is again evident that the proposed technique exhibits high accuracy for the volume measurement.

Table IV gives the surface-areas computed based on the images. The DB eggs have the highest deviation of $4.01 \mathrm{~cm}^{2}$.
This is also believed due to the size variation of this group of eggs.

TABLE III. MEASURED VOLUME

\begin{tabular}{|c|c|c|c|c|c|c|c|}
\hline \multirow[b]{2}{*}{$\begin{array}{l}\text { Egg } \\
\text { Type }\end{array}$} & \multirow[b]{2}{*}{ Size } & \multirow[b]{2}{*}{$\begin{array}{r}V_{-P M} \\
\left(\mathrm{~cm}^{3}\right)\end{array}$} & \multirow[b]{2}{*}{$\begin{array}{r}V_{-I M} \\
\left(\mathrm{~cm}^{3}\right) \\
\end{array}$} & \multirow[b]{2}{*}{$\begin{array}{l}\text { RE } \\
(\%)\end{array}$} & \multicolumn{3}{|c|}{ Standard Deviation } \\
\hline & & & & & $\begin{array}{l}V_{-P M} \\
\left(\mathrm{~cm}^{3}\right)\end{array}$ & $\begin{array}{c}V_{-I M} \\
\left(\mathrm{~cm}^{3}\right)\end{array}$ & $\begin{array}{l}\text { RE } \\
(\%)\end{array}$ \\
\hline \multirow{3}{*}{ FR } & $\mathrm{M}_{\mathrm{f}}$ & 53.83 & 53.86 & 0.06 & 2.27 & 2.34 & 0.76 \\
\hline & $\mathrm{L}_{\mathrm{f}}$ & 60.73 & 60.54 & -0.32 & 2.37 & 2.37 & 0.51 \\
\hline & $\mathrm{XL}_{\mathrm{f}}$ & 76.01 & 76.13 & 0.14 & 3.95 & 4.37 & 0.86 \\
\hline $\mathrm{C}$ & $\mathrm{M}_{\mathrm{C}}$ & 61.34 & 61.63 & 0.47 & 0.24 & 0.47 & 0.82 \\
\hline B & $\mathrm{M}_{\mathrm{B}}$ & 57.14 & 57.02 & -0.20 & 5.71 & 5.66 & 0.76 \\
\hline DB & $\mathrm{M}_{\mathrm{D}}$ & 62.56 & 62.61 & 0.08 & 7.04 & 7.15 & 0.59 \\
\hline
\end{tabular}

TABLE IV. SURFACE AREA

\begin{tabular}{|c|c|c|c|}
\hline \multirow{2}{*}{ Egg Type } & Size & $\left.\mathbf{S} \mathbf{( c m}^{\mathbf{2}}\right)$ & $\begin{array}{c}\text { Standard Deviation } \\
\left(\mathbf{c m}^{\mathbf{2}}\right)\end{array}$ \\
\hline \multirow{2}{*}{$\mathrm{FR}$} & $\mathrm{M}_{\mathrm{f}}$ & 73.01 & 1.83 \\
\cline { 2 - 4 } & $\mathrm{L}_{\mathrm{f}}$ & 77.32 & 1.24 \\
\cline { 2 - 4 } & $\mathrm{XL}_{\mathrm{f}}$ & 84.98 & 2.26 \\
\hline $\mathrm{C}$ & $\mathrm{M}_{\mathrm{C}}$ & 77.42 & 2.25 \\
\hline $\mathrm{B}$ & $\mathrm{M}_{\mathrm{B}}$ & 75.72 & 3.45 \\
\hline $\mathrm{DB}$ & $\mathrm{M}_{\mathrm{D}}$ & 78.90 & 4.01 \\
\hline
\end{tabular}

\section{Accuracy and Uncertainty of the Measurement}

To assess the accuracy and uncertainty of the measurement of the system, two reference objects, e.g., a table tennis ball and a squash ball, were used, as shown in Fig. 8. The diameters of the balls were measured using a Vernier caliper, and the values of the surface areas were then computed using the sphere surface-area formula, as well as the proposed imaging technique. Table $\mathrm{V}$ summarizes the measured and computed results. As can be seen, the surface areas computed based on the imaging approach are very close to that based on the mathematic formulae. This validates that the proposed method is capable of measuring the geometric parameters of a given object accurately.

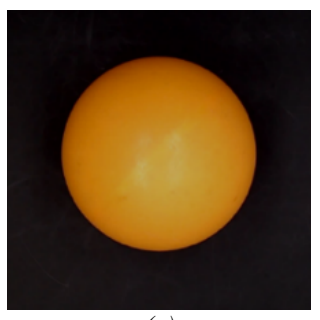

(a)

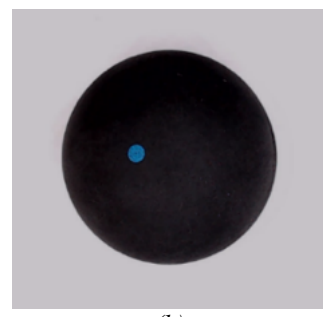

(b)
Fig. 8. (a) Table tennis ball, and (b) Squash ball.

TABLE V. SURFACE AREA OF THE REFERENCE OBJECTS.

\begin{tabular}{|c|c|c|c|c|}
\hline Reference & $\begin{array}{c}\text { Radius } \\
(\mathbf{c m})\end{array}$ & $\begin{array}{c}\mathbf{S}_{-} \text {PM } \\
\left(\mathbf{c m}^{2}\right)\end{array}$ & $\begin{array}{c}\mathbf{S}_{-} \text {IM } \\
\left(\mathbf{c m}^{2}\right)\end{array}$ & RE (\%) \\
\hline $\begin{array}{c}\text { Table tennis } \\
\text { ball }\end{array}$ & 2.00 & 50.27 & 49.65 & -1.23 \\
\hline $\begin{array}{c}\text { Squash } \\
\text { ball }\end{array}$ & 2.15 & 58.08 & 57.12 & -1.65 \\
\hline
\end{tabular}

It is worth to note that there are possible sources that may introduce the errors and uncertainty in the measurements. Firstly, there is an inherent difference between the working principles. Secondly, the perspective effect along the optical path of the camera could cause the small variations of the 
length and area conversion coefficients across the 2-D images of the eggs considering that eggs vary in sizes. However, the level of the errors is considered to be within an acceptable level.

\section{CONCLUSION}

A digital imaging based method for the real-time 3-D reconstruction and characterization of avian eggs is presented. Two low-cost cameras are used to acquire the images of the eggs under well control lighting conditions. The images are binarized and the egg regions are segmented in the CMYK color space. The length and breadth of the egg are then extracted from the images for reconstructing the 3-D model of the egg. A set of geometrical parameters including the maximum length, breadth, volume and surface area are finally derived from the 3-D model of the egg. The proposed method has been examined for different types and sizes of avian eggs. The effectiveness of the method has also been evaluated using standard table tennis and squash balls as references. The results have suggested that the proposed method is accurate and reliable for performing the 3-D reconstruction and measurements of avian eggs with an accuracy of $98 \%$ in comparison to the reference measurements. The proposed method has been proven to be a practical, accurate and reliable means for the real-time measurement of avian eggs not only for the poultry industry but also in ornithological and zoological studies.

\section{REFERENCES}

[1] V. G. Narushin, and M. N. Romanov, "Egg physical characteristics and hatchability," World's Poult. Sci. J., vol. 58, no. 03, pp. 297-303, 2002 .

[2] V. G. Narushin, V. P. Bogatyr, and M. N. Romanov, "Relationship between hatchability and non-destructive physical measurements of chicken eggs," J. Agric. Sci., vol. 154, no. 2, pp. 359-365, 2016.

[3] J. Rendel, "Variations in the weights of hatched and unhatched ducks' eggs," Biometrika, vol. 33, no. 1, pp. 48-58, 1943.

[4] C. C. Brunson, and G. F. Godfrey, "The Relationship of Egg Shape, Egg Weight, Specific Gravity and 21-Day Incubation Weight-Loss to Hatchability of Broad-Breasted Bronze Turkey Eggs," Poult. Sci., vol. 32 , no. 5, pp. 846-849, 1953.
[5] W. V. Reid, and P. D. Boersma, "Parental quality and selection on egg size in the magellanic penguin," Evolution, vol. 44, no. 7, pp. 1780-1786, 1990.

[6] P. D. Boersma, and G. A. Rebstock, "Calculating egg volume when shape differs: When are equations appropriate?," J. F. Ornithol., vol. 81, no. 4, pp. 442-448, 2010.

[7] D. F. Hoyt, "Practical methods of estimating volume and fresh weight of bird eggs," The Auk, pp. 73-77, January 1979.

[8] T. Y. Wang, and S. K. Nguang, "Low cost sensor for volume and surface area computation of axi-symmetric agricultural products," J. Food Eng., vol. 79, no. 3, pp. 870-877, 2007.

[9] V. G. Narushin, "Egg geometry calculation using the measurement of length and breadth," Poult. Sci. vol. 84, no. 3, pp. 482-484, 2005.

[10] A. J. Romanoff, and A.L. Romanoff, “The Avian Egg,” John Wiley, New York, 1949.

[11] P. Zhou, W. Zheng, C. Zhao, C. Shen, and G. Sun, "Egg volume and surface area calculations based on machine vision," Computer Computing Technol. in Agriculture II, Vol. 3, vol. 295, pp. $1647-$ 1653,2009

[12] O. C. Ting, D.D. Lichti, A. Jahraus, H. Esfandiari, H. Lahamy, J. Steward, and M. Glanzer, "An egg volume measurement system based on the Microsoft Kinect," Sensors, vol. 18, no. 8, 2454, 2018.

[13] J. Troscianko, "A simple tool for calculating egg shape, volume and surface area from digital images," Int. J. Avian Sci., vol. 156, pp. 874-878, 2014.

[14] M. Z. Zalhan, S. Sera Syarmila, I. Mohd Nazri, and I. Mohd Taha, "Vision-based egg grade classifier," 2016 Int. Conf. Info. Commun. Technol., Kuala Lumpur, Malaysia, 16-17 May 2016.

[15] J. Siswantoro, A. S. Prabuwono, and A. Abdullah, "Volume measurement algorithm for food product with irregular shape using computer vision based on Monte Carlo method," J. ICT Res. Appl., vol. 8 , no. 1, pp. 1-17, 2014.

[16] W. Zhang, X. Wu, Z. Qiu, and Y. He, “A novel method for measuring the volume and surface area of egg," J. Food Eng., vol. 170, pp. 160$169,2016$.

[17] M. S. Ibrahim, A. A. Badr, M. R. Abdallah and I. F. Eissa, "Bounding box object localization based on image superpixelization," Procedia Computer Sci., vol. 13, pp. 108-119, 2012.

[18] E. S. Bridge, R. K. Boughton, R. A. Aldredge, T. J. E. Harrison, R. Bowman, and S. J. Schoech, "Measuring egg size using digital photography: Testing Hoyt's method using Florida Scrub-Jay eggs," J. F. Ornithol., vol. 78, no. 1, pp. 109-116, 2007.

[19] S. W. Hughes, "Archimedes revisited: A faster, better, cheaper method of accurately measuring the volume of small objects," Phys. Educ., vol. 40, no. 5, pp. 468-474, 2005. 